\title{
Filters in Dissolution Testing: Evaluation and Selection
}

\author{
Janet Smith, Jesmi George, Timothy Nadler, and Vivek Joshi*
}

EMD Millipore Corp., Burlington, MA, USA.

e-mail:Vivek.joshi@milliporesigma.com

\section{ABSTRACT}

Filtration is critical in drug dissolution testing as filtration stops the dissolution process and allows for accurate quantitation through separation of dissolved and un-dissolved components. Filtration is one of the simplest sample preparation techniques. Regardless, little attention is paid to filter selection, and that can negatively impact the quantitation of dissolved active pharmaceutical ingredients. This review provides information on various characteristics of membranes and filter devices to help researchers make an educated decision on filter selection during the method development and validation phases.

KEYWORDS: Membrane filtration, dissolution testing, extractables, analyte binding, pore size

\section{INTRODUCTION}

The ultimate objective of dissolution testing for oral dosage forms is to ensure adequate and reproducible bioavailability of the active pharmaceutical ingredient (API). The purpose of dissolution testing as described in United States Pharmacopeia (USP) monographs is to obtain drug release characteristics of a formulation or a batch of formulation under the experimental conditions. Compliance with the test indicates that the API will be soluble in the media within a reasonable timeframe in an in vitro setting.

A typical dissolution test workflow starts with preparing the dissolution medium that is appropriate for the formulation. One thing to keep in mind regarding the dissolution medium is the impact of oxygen dissolved in it because oxygen can affect dissolution of the API. Hence, it is important to deaerate the medium before the dissolution test is performed $(1,2)$. Filtration of media through membranes under a vacuum is one of the methods used to deaerate dissolution media, and membrane flow characteristics can play an important role in this context. After the medium is prepared, the actual dissolution test is carried out with the formulation under study. Samples are collected at various time points for analysis.

One of the most critical steps in the dissolution testing of pharmaceutical dosage formulations is the filtration of the samples withdrawn from the dissolution apparatus.
Even the USP monographs on dissolution testing stress that the sample analysis is to be carried out on a filtered sample. The dissolution sample may contain undissolved active ingredient(s) and excipients at any given time during the test. It is therefore critical to filter the sample before any downstream analysis can be conducted (3). Typically, filter frits, canula filters, and membrane-based syringe filters are used for sample filtration. Given that highperformance liquid chromatography (HPLC) or ultra HPLC (UHPLC) are the most common methods of downstream analysis following these tests, membrane-based filters are most commonly used after the dissolution test. Filtration is one of the simplest sample preparation techniques; however, there are quite a few misconceptions about the choice of membrane-based filters. Owing to the simplicity of filtration, sometimes insufficient consideration is given to filter selection in dissolution testing. Many times, researchers select a membrane filter that is either stocked or previously used in the laboratory. In this review article, we present data on various properties of membranes such as pore size, pore size distribution, analyte binding, and extractables. The impact of these characteristics on dissolution testing and quantitative analysis is presented.

\section{METHODS}

We searched for literature on membrane filters and their characteristics that was published within the last 20 years. Because membrane filtration is a simple technique, it was hard to find a large body of literature on membrane characteristics. The majority of data presented in this

* Corresponding author. 
article were generated in house for the purpose of this review. The websites of various manufacturers of membrane filters also provided useful information about membranes and their characteristics.

\section{CHARACTERISTICS OF MEMBRANE FILTERS}

Membrane filters are available in a wide variety for sample filtration in dissolution testing. It is the membranes themselves that help to separate the dissolved components of a formulation (active ingredient and excipients) from the undissolved components. The membrane is the key component of these filters; therefore, a better understanding of membrane characteristics is important to aid in the selection of the right membrane for an application.

Table 1 lists commonly used membranes in dissolution testing and their manufacturing methods as well as some advantages and disadvantages.

\section{Pore Size, Pore Morphology, and Percentage of Porosity}

The different ways in which membranes are manufactured result in differences in their pore structure and morphology. Table 1 shows scanning electron micrographs of some of the commonly used membranes for filtration application. Membranes manufactured using either immersion casting or air casting show a random pore structure, creating a tortuous filtration path within the membrane. Depending on manufacturing technique, pore asymmetry can also be created in these structures whereby membrane pore size changes throughout the filtration path. Such asymmetric membranes can show better flow characteristics compared to symmetric membranes. On the other hand, a polytetrafluoroethylene (PTFE) membrane, which is manufactured using the expansion technique, clearly shows pores that are elongated in the direction of the expansion. Finally, a polycarbonate membrane shows perfectly spherical pores which extend through the thickness of the membrane, creating cylindrical pores within the membrane. Of all the membranes included in Table 1, polycarbonate membranes have the least number of pores (5-20\% porosity), which significantly limits flow rate. All other membranes have very high levels of porosity (60-80\%) which improves flow characteristics. The pore structure and morphology of membranes also impact particle retention and filtration flow rate, among other characteristics $(4,5)$.

Given that membranes from different manufacturers are made and tested differently, there can be variations in particle retention characteristics. Figure 1 shows the particle retention of four syringe filters that contain different membranes and were manufactured by two different vendors. Of the $0.2-\mu \mathrm{m}$ filters tested, those from vendor $A$ (containing polyether sulfone [PES], nylon, polyvinylidene fluoride [PVDF], and hydrophilic [PTFE] membranes) show quantitative retention of $0.3-\mu \mathrm{m}$ latex beads, whereas two filters from vendor B (containing PVDF and polypropylene membranes) only retained about $80-85 \%$ of the particles, allowing about $15-20 \%$ to pass into the filtrate.

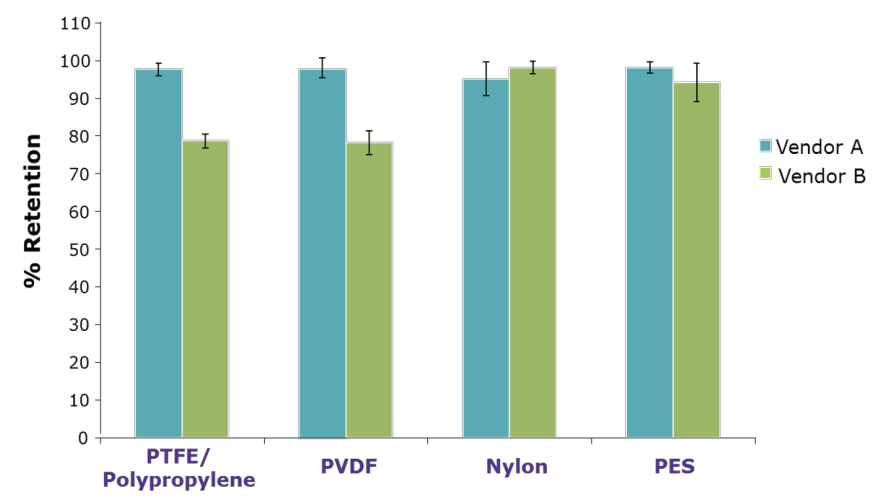

Figure 1. Particle retention behavior of various syringe filters. A suspension of 0.3- $\mu \mathrm{m}$ Latex beads in water was filtered through $0.2-\mu \mathrm{m}$ pore size syringe filters and filtrate was collected. Filtrate and the original suspension were analyzed using UV spectrometry to determine

concentration of particles and thereby particle retention by these syringe filters.

PTFE: polytetrafluoroethylene; PVDF: polyvinylidene fluoride; PES: polyether sulfone.

The impact of these un-retained particles in the filtrate can also be seen in the back-pressure measurements from UHPLC analysis (data not shown). When the hydrophilic PTFE membrane from vendor A $(0.2$ and $0.45 \mu \mathrm{m})$ were compared with polypropylene membranes from vendor $B$ (same size), an increase in back pressure was observed for the mobile phase filtered through the polypropylene membrane (1500-2000 PSI). The hydrophilic PTFE membrane retained particles quantitatively; no increase in backpressure was observed as expected.

\section{Chemical Compatibility}

Another key characteristic of membranes and syringe filter devices is their chemical compatibility. Of the housing materials used for syringe filters, high density polyethylene (HDPE) and polypropylene (PP) are resistant to a wide variety of chemicals used in dissolution testing and in HPLC and UHPLC analysis. Syringe filters with housings composed of acrylic resin and poly vinyl chloride (PVC) are also available. These housing materials are compatible with aqueous solutions, but their compatibility with solvents is limited. The membranes that are commonly available and compatible with 
Table 1. Key Characteristics of Various Microporous Membranes Used in Pharmaceutical Quality Control Testing

\begin{tabular}{|c|c|c|c|c|}
\hline Membrane & Manufacturing Method & Advantages & Disadvantages & Pore Morphology \\
\hline Hydrophilic PTFE & Heat and Expansion & $\begin{array}{l}\text { - Compatible with } \\
\text { aqueous and organic } \\
\text { solutions } \\
\text { - Low extractables } \\
\text { - Low binding to small } \\
\text { molecule APIs }\end{array}$ & $\begin{array}{l}\text { - Low to medium } \\
\text { protein binding }\end{array}$ & 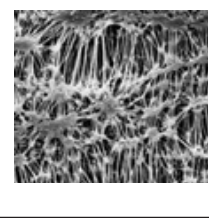 \\
\hline Hydrophobic PTFE & Heat and Expansion & $\begin{array}{l}\text { - Compatible with } \\
\text { broad range of organic } \\
\text { solutions }\end{array}$ & $\begin{array}{l}\text { - Not suitable for } \\
\text { dissolution testing }\end{array}$ & 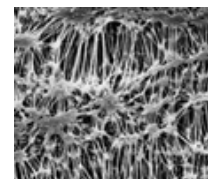 \\
\hline Hydrophilic PVDF & Immersion casting & $\begin{array}{l}\text { - Compatible with } \\
\text { aqueous solutions } \\
\text { - Low extractables } \\
\text { - Very low binding to } \\
\text { protein analytes }\end{array}$ & $\begin{array}{l}\text { - Limited compatibility } \\
\text { with organic solvents }\end{array}$ & \\
\hline Nylon & Immersion casting & $\begin{array}{l}\text { - Compatible with } \\
\text { aqueous and organic } \\
\text { solutions }\end{array}$ & $\begin{array}{l}\text { - High binding of } \\
\text { analytes } \\
\text { - Susceptible to } \\
\text { hydrolysis at low or } \\
\text { high pH }\end{array}$ & \\
\hline Polyether sulfone (PES) & Immersion casting & $\begin{array}{l}\text { - Compatible with } \\
\text { aqueous solutions } \\
\text { - Low protein }\end{array}$ & $\begin{array}{l}\text { - Limited compatibility } \\
\text { with organic solvents }\end{array}$ & \\
\hline Regenerated Cellulose & Immersion casting & $\begin{array}{l}\text { - Compatible with } \\
\text { aqueous solutions } \\
\text { - Low extractables } \\
\text { - Low binding }\end{array}$ & $\begin{array}{l}\text { - Limited compatibility } \\
\text { with organic solvents }\end{array}$ & NA \\
\hline Mixed cellulose ester (MCE) & Air casting & $\begin{array}{l}\text { - Compatibile with } \\
\text { aqueous solutions }\end{array}$ & $\begin{array}{l}\text { - Limited compatilbility } \\
\text { with organic solvents } \\
\text { - High protein binding }\end{array}$ & \\
\hline Polycarbonate & $\begin{array}{c}\text { E beam bombardment } \\
\text { followed by chemical } \\
\text { etching }\end{array}$ & $\begin{array}{l}\text { - Cylindrical pores with } \\
\text { absolute pore size } \\
\text { - Smooth/glassy } \\
\text { surface suitable for } \\
\text { microscopy }\end{array}$ & $\begin{array}{l}\text { - Low porosity } \\
\text { - Low flow rate } \\
\text { - Limited mechanical } \\
\text { strength }\end{array}$ & $\therefore$ \\
\hline Polypropylene & Melt casting & $\begin{array}{l}\text { - Compatible with } \\
\text { aqueous and organic } \\
\text { solutions } \\
\text { - Low extractables }\end{array}$ & $\begin{array}{l}\text { - Low to medium } \\
\text { protein binding }\end{array}$ & NA \\
\hline
\end{tabular}

API: active pharmaceutical ingredient; PTFE: polytetrafluoroethylene; PVDF: polyvinylidene fluoride; NA: not applicable. 
most chemicals used in dissolution procedures are hydrophilic PTFE and polypropylene membranes. All the other membranes have limited compatibility with certain solutions and solvents, and hence should be selected based on the solution being filtered. Because most solutions filtered during dissolution testing are aqueous, hydrophilic membranes are typically used. In the case of alcohol dose dumping studies performed on pharmaceutical formulations, it is important to keep in mind that, in these studies, the membrane needs to be compatible with hydroalcoholic dissolution media (6).

\section{Filtration Device Designs}

Even though membranes can be used directly for filtration of dissolution media and chromatographic mobile phase, filtration for actual samples requires that a membrane be present in a device. Table 2 shows various filtration device formats along with their advantages and disadvantages.

The most common filtration format used in dissolution testing is the syringe filter. These are available with a wide range of membranes, pore sizes, and filter sizes. Depending on the design, syringe filters may be compatible with automated filtration systems. The main disadvantage of syringe filters is that the filtration process is manual and needs to be done one at a time, which can be challenging when filtering many samples. Recently, there have been innovations, such as vacuum-based filtration systems that allow the use of standard syringe filters and the filtration of multiple samples simultaneously.
Another common format for filtration is the frit, or canula filter, which filters the sample as it is being collected. This type of filter is also compatible with automated sample collection and filtration systems. The main disadvantage of this filter type is pore size, which is generally large (1$10 \mu \mathrm{m})$. Samples filtered through these devices may be suitable for analysis by ultraviolet (UV) spectroscopy, but they are not suitable for HPLC or UHPLC analysis.

The remaining filtration systems are less commonly used in dissolution testing because they are not suitable for routine dissolution workflow. Some of the examples of these filtration formats are syringeless membrane filters, multiwell plates, and devices containing ultrafiltration membranes.

\section{CHARACTERISTICS OF SYRINGE FILTERS THAT IMPACT QUANTITATIVE PROCEDURES}

To quantitate the amount of active ingredient dissolved in a dissolution medium, UV spectrophotometry or HPLC/UHPLC coupled with UV detection are commonly used. These quantitative procedures can be negatively impacted by two main problems that syringe filters pose: 1) syringe filters can add contaminants (extractables) to the filtrate, and 2) syringe filters can bind the analyte of interest, leading to incorrect quantitation.

\section{Extractables}

Extractables are low molecular weight compounds that dissolve in the sample or solvent that is being

Table 2. Various Device Formats for Sample Filtration Following Dissolution Testing

\begin{tabular}{|c|c|c|}
\hline Filtration Format & Pros & Cons \\
\hline Syringe Filters & $\begin{array}{l}\text { - Most common filtration format in dissolution testing } \\
\text { - Various membranes, pore sizes, housing materials \& } \\
\text { volume capability } \\
\text { - Compatible with downstream HPLC/UHPLC } \\
\text { - Compatible with automation }\end{array}$ & $\begin{array}{l}\text { - Filtration is manual process } \\
\text { - Automated filter changing stations can be expensive and } \\
\text { not compatible with all syringe filters }\end{array}$ \\
\hline Frit / Canula Filters & $\begin{array}{l}\text { - Compatible with automated filtration } \\
\text { - Reusable multiple times during a single experiment } \\
\text { - Sample is filtered as it is collected }\end{array}$ & $\begin{array}{l}\text { - Pore size is generally large }(1-10 \mu \mathrm{m}) \\
\text { - Filtered sample may not be suitable for HPLC/UHPLC }\end{array}$ \\
\hline $\begin{array}{c}\text { Syringeless } \\
\text { Membrane Filters }\end{array}$ & - Sample is filtered directly in HPLC vial & $\begin{array}{l}\text { - Limited sample volume (Typical less than } 2 \mathrm{~mL} \text { ) } \\
\text { - Difficult to flush a certain sample volume to reduce } \\
\text { analyte binding and extractables }\end{array}$ \\
\hline $\begin{array}{l}\text { Membrane-Based } \\
\text { Multiwell Plates }\end{array}$ & - High throughout sample filtration & $\begin{array}{l}\text { - Processing volume }(0.3-1.5 \mathrm{~mL}) \text { too small for dissolution } \\
\text { testing } \\
\text { - Number of samples insufficient to fully utilize a plate }\end{array}$ \\
\hline $\begin{array}{l}\text { Ultrafiltration } \\
\text { Devices }\end{array}$ & - Useful when dealing with viscous dissolution medium & $\begin{array}{l}\text { - Limited membrane options available } \\
\text { - Generally, these products are only campatible with } \\
\text { aqueous solutions }\end{array}$ \\
\hline
\end{tabular}

HPLC: high performance liquid chromatography; UHPLC: ultra-HPLC. 
filtered through a membrane. Extractables manifest as contaminants in the quantitative procedure $(7,8)$.

If the impurity peak from the extractable co-elutes with the analyte of interest, it causes inaccurate and variable results, because extractables can vary from sample to sample. On the other hand, if the extractable impurities elute far from the analyte of interest, quantitation of analyte may not be impacted. Still, the presence of these extraneous peaks needs to be explained. Generally, when contaminant peaks are greater than $1 \%$ of the analyte peak observed in a chromatogram, these peaks need to be reported and identified; extractable peaks that are well separated from analyte of interest also need to be identified.

Ideally in these cases, when selecting a filter, one that is designed not to release any extractables is preferred. However, as any surface coming in contact with the sample can release impurities into the sample, an HPLC-certified low-extractables filter should be used for dissolution testing. Most filtration system vendors provide syringe filters that are certified for low extractable impurities using various solvents and chromatographic conditions; however, they need to be evaluated, case by case.

Figure 2 shows the profile of extractables in various syringe filters, as determined by HPLC-UV analysis. It is interesting to note that all these filters are certified as having low extractables based on HPLC analysis. From the three types of syringe filters we tested, hydrophilic PTFE membrane-based syringe filters have the lowest amount of extractables. Both polypropylene and nylon membrane-based syringe filters show high levels of extractables. As can be seen, both of these syringe filters show extractable peaks that elute in regions of the chromatogram where the analyte of interest is expected to elute, thus interfering with its quantitation. Depending on the extraction solvent or dissolution medium, it is possible that these filters will have a different profile of extractables. It is recommended to evaluate the extractables profile of all filters under consideration during method development. As can be clearly seen in Figure 2, not all HPLC-certified syringe filters are identical; they can have variable levels of extractables.

Nylon membrane-based syringe filters are commonly used in dissolution testing, but they may contaminate the sample with extractable impurities. It is generally believed that the extractables present in nylon membranes usually consist of low molecular weight polymeric impurities (9).
Use of liquid chromatography and mass spectrometry (LC$M S$ ) is not prevalent in dissolution testing, but within the pharmaceutical analysis field, LC-MS is very commonly used due to its sensitivity and specificity. Given that most of the commercially available syringe filters are certified for low extractables by HPLC-UV analysis, if LC-MS is used in downstream analysis, additional extractables testing should be performed to ensure that the filtrate does not contain impurities that can interfere in mass spectral analysis.

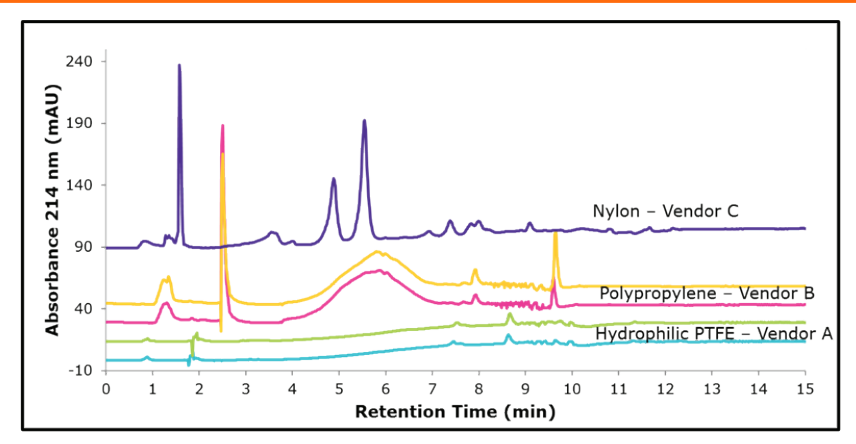

Figure 2. Extractables from various syringe filters. Extractables testing from syringe filters was carried out using $70 \%$ acetonitrile in water as extraction solvent. Three different HPLC-certified syringe filters from three different vendors were used. Extraction solvent $(1 \mathrm{~mL})$ was filtered through the syringe filters, and resulting filtrate was analyzed using reversed phase chromatography and a C-18 column. Water and acetonitrile were used as mobile phase components and a $0-100 \%$ Acetonitrile gradient was used to evaluate extractables profile from these syringe filters; $214 \mathrm{~nm}$ was used as the detection wavelength to ensure detection of most extractable impurities.

PTFE: polytetrafluoroethylene; HPLC: high-performance liquid chromatography.

\section{Analyte Binding}

Similar to extractables, analyte binding is almost impossible to fully eliminate, so the strategy is to minimize analyte binding to any surface that the sample may contact. One should consider that syringe filters for dissolution testing come into contact with the sample just before it is analyzed, but syringes, vials, or any other surface that can come in contact with a sample, including chromatographic columns, can potentially bind to the analyte of interest (10-14). The main causes for analyte binding to surfaces are weak secondary interactions such as hydrogen bonding, hydrophobic interactions, and electrostatic interactions.

Table 3 shows the percentage of acetaminophen binding to syringe filters containing various types of membranes (glass fiber, hydrophilic PTFE, hydrophilic PVDF, nylon, and PES). Of all the membranes tested in this study, nylon membranes show the highest level of binding to acetaminophen. 
In general, nylon membranes show much higher binding for certain analytes when compared to PTFE and PVDF membranes. This binding characteristic is inherent to nylon membranes. The high level of binding is due to electrostatic as well as ionic interactions. Nylon membranes contain amino and carboxylate functional groups that may form strong ionic bonds with various analytes, many of which are ionizable in nature. Nylon membranes also form very strong hydrogen bonds with various analytes. Many pharmaceuticals are basic by nature with functional groups that can ionize under appropriate conditions, thus exacerbating the binding effect with nylon.

As can be clearly seen from Table 3, analyte binding can be reduced as more sample is filtered; as the filter surface becomes fully saturated, there is less analyte loss due to binding. This is a strategy that is commonly used in dissolution testing, wherein a certain volume of solution is discarded before the sample is collected for analysis. Typically, the discarded volume is between 3 and $10 \mathrm{~mL}$, but this strategy to be evaluated during method development, on a case-by-case basis.

For multicomponent pharmaceuticals, the binding behavior of different analytes within the formulation varies, because binding is dependent on the physicochemical characteristics of the membrane and analyte. Table 4 shows binding behavior in a multicomponent migraine formulation containing three different APIs.

Table 3. Acetaminophen Binding to Various Membranes

\begin{tabular}{|c|c|c|c|c|c|}
\hline Filtrate & Glass Fiber & Hydrophilic PVDF & Nylon & PES & Hydrophilic PTFE \\
\hline $1 \mathrm{~mL}$ & $98 \%$ & $96 \%$ & $74 \%$ & $98 \%$ & $96 \%$ \\
\hline $2 \mathrm{~mL}$ & $99 \%$ & $98 \%$ & $100 \%$ & $100 \%$ & $98 \%$ \\
\hline $3 \mathrm{~mL}$ & $99 \%$ & $98 \%$ & $102 \%$ & $101 \%$ & $103 \%$ \\
\hline $4 \mathrm{~mL}$ & $99 \%$ & $100 \%$ & $101 \%$ & $102 \%$ & $98 \%$ \\
\hline $5 \mathrm{~mL}$ & $101 \%$ & $101 \%$ & $102 \%$ & $102 \%$ & $98 \%$ \\
\hline $10 \mathrm{~mL}$ & $100 \%$ & $102 \%$ & $102 \%$ & $101 \%$ \\
\hline
\end{tabular}

Note - This analyte binding study for acetaminophen was carried out under dissolution test conditions for 240 min. Simulated gastric fluid without enzymes was used as dissolution medium. After dissolution test was completed, the sample was filtered using various syringe filters containing membranes listed in the table and fractions of filtrate were collected. The fractions were analyzed using UV spectroscopy at 243 nm. Similar to filtration, the sample was also centrifuged and the supernatant was collected for analysis. This provided a standard for $100 \%$ API recovery (no binding). API recovery from filtered samples was calculated using centrifuged sample as a standard.

PVDF: polyvinylidene fluoride; PES: polyether sulfone; PTFE: polytetrafluoroethylene; API: active pharmaceutical ingredient.

\begin{tabular}{|c|c|c|c|c|}
\hline \multirow[t]{2}{*}{ Analyte of Interest } & \multirow[t]{2}{*}{ Filtrate $(\mathrm{mL})$} & \multicolumn{3}{|c|}{ Syringe Filter Used } \\
\hline & & Hydrophilic PTFE & Nylon & PVDF \\
\hline \multirow[t]{4}{*}{ Acetaminophen } & $1^{\text {st }} \mathrm{mL}$ & 110.4 & 58.4 & 109.0 \\
\hline & $2^{\text {nd }} \mathrm{mL}$ & 112.3 & 109.3 & 110.6 \\
\hline & $3^{\text {rd }} \mathrm{mL}$ & 112.2 & 111.3 & 110.8 \\
\hline & $5^{\text {th }} \mathrm{mL}$ & 112.2 & 111.5 & 110.7 \\
\hline \multirow[t]{4}{*}{ Acetyl Salicylic Acid } & $1^{\text {st }} \mathrm{mL}$ & 119.5 & 28.2 & 117.4 \\
\hline & $2^{\text {nd }} \mathrm{mL}$ & 121.4 & 116.1 & 119.0 \\
\hline & $3^{\text {rd }} \mathrm{mL}$ & 121.2 & 121.6 & 119.1 \\
\hline & $5^{\text {th }} \mathrm{mL}$ & 121.3 & 122.0 & 119.2 \\
\hline \multirow[t]{4}{*}{ Caffeine } & $1^{\text {st }} \mathrm{mL}$ & 114.2 & 106.0 & 112.2 \\
\hline & $2^{\text {nd }} \mathrm{mL}$ & 113.9 & 112.6 & 112.6 \\
\hline & $3^{\text {rd }} \mathrm{mL}$ & 113.8 & 112.9 & 112.7 \\
\hline & $5^{\text {th }} \mathrm{mL}$ & 113.6 & 112.9 & 112.7 \\
\hline
\end{tabular}

Note - The dissolution testing of the multicomponent migraine tablet formulation was conducted using $900 \mathrm{~mL}$ of water as dissolution media, in USP dissolution apparatus 2 at $100 \mathrm{rpm}$. Samples were collected after dissolution test and filtered through three different syringe filters containing hydrophilic PTFE, nylon and hydrophilic PVDF membranes. Various fractions of filtrate were collected $\left(1^{\text {st }}, 2^{\text {nd }}, 3^{\text {rd }}\right.$, and $\left.5^{\text {th }}\right)$ and analyzed using UHPLC with UV detection at $275 \mathrm{~nm}$. API recovery was calculated using UHPLC peak areas for filtered sample, individual compound standard, and the label claim for the tablet. PTFE: polytetrafluoroethylene; PVDF: polyvinylidene fluoride; UHPLC: ultra-high-performance liquid chromatography; API: active pharmaceutical ingredient. 
Both acetaminophen and aspirin show strong binding to the nylon membrane (when no volume is discarded), whereas caffeine does not show any binding to nylon.

For oral formulations and dissolution testing, small molecule binding characteristics for membranes are important, but if protein binding characteristics of membranes are also important, then similar studies should be done with different membranes. In general, hydrophilic PVDF shows the lowest amount of analyte loss due to protein binding, whereas mixed cellulose, ester, and nylon membranes tend to show higher protein binding (data not shown).

\section{DISCUSSION}

This review has examined membrane characteristics and provided guidance for filter selection for use in pharmaceutical quality control (QC) testing. As scientists develop and validate dissolution methods for oral formulations, it is important to understand the impact of membrane characteristics on formulations and on the analyte(s) of interest. A membrane filter that worked for previous formulations may not necessarily work for new formulations. In essence, this article provides a blueprint for filter selection and validation studies as part of method development.

For a sample to be suitable for chromatography, the sample must be soluble in a solvent and free of particulate impurities. Filtration is one of the simplest sample preparation techniques used to remove particulate impurities. Particle removal efficacy of membrane filters is dependent on the size and morphology of the pores contained in the membrane, as well as the design and features of the filtration device. As membranes filters remove particles, they can also remove some of the analyte owing to analyte binding. The membrane filter can also add extractable impurities to the sample (15). Both phenomena can impact accurate quantitation of the analyte of interest.

In the future, we will examine analyte binding in greater detail, as binding has the largest impact on quantitation. The effect of analyte concentration and the differences in sample processing techniques as well as the physicochemical characteristics of the analyte and dissolution media will be evaluated.

\section{CONCLUSIONS}

Filtration is a critical step in any dissolution procedure, as it stops the dissolution process and removes all undissolved materials present in the sample. Despite the fact that filtration is a very simple sample preparation technique, it remains the least understood by researchers. This paper shows the importance of various parameters when selecting filters for dissolution testing, and how they impact the quantitative determination of the amount of active ingredient dissolved.

\section{CONFLICTS OF INTEREST}

The authors disclosed no conflicts of interest related to this article.

\section{REFERENCES}

1. Fliszar, K. A.; Forsyth, R. J.; Li, Z.; Martin, G. P. Effect of dissolved gases in surfactant dissolution media. Dissolut. Technol. 2005, 12, 6-10. 12, 6-10. DOI: 10.14227/DT120305P6.

2. Degenhardt O. S.; Waters B.; Rebelo-Cameirao A.; Meyer A.; Brunner H.; Toltl N. P. Comparison of the effectiveness of various deaeration techniques. Dissolut. Technol., 2004, 11, 6-11. DOI: 10.14227/DT110104P6.

3. Joshi V.; Blodgett J.; George J.; Brinker J. Impact of sample preparation on dissolution testing: Drug binding and extractable impurities and their effect on dissolution data. Dissolut. Technol. 2008, 15, 20-27. DOI: 10.14227/DT150408P20.

4. Wang R.; Liu Y.; Li B.; Hsiao B. S.; Chu B. Electrospun nanofibrous membranes for high flux microfiltration, J. Membr. Sci. 2012, 392-393, 167-174. DOI: 10.1016/j.memsci.2011.12.019.

5. Liu Y.; Wang R.; Ma H.; Hsiao B. S.; Chu B. High flux microfiltration filters based on electrospun polyvinylalcohol nanofibrous membranes, Polymer (Guildf.) 2013, 54, 548-556. DOI: 10.1016/j.polymer.2012.11.064.

6. Anand O.; Yu L. X.; Conner D. P.; Davit B. M. Dissolution testing for generic drugs: an FDA perspective. AAPS J. 2011, 13, 328335. DOI: 10.1208/s12248-011-9272-y.

7. Huang M.; Horwitz T. S.; Zweiben C.; Singh S. K. Impact of extractables/leachables from filters on stability of protein formulations. J. Pharm. Sci. 2011, 100, 4617-4630. DOI: 10.1002/jps.22670.

8. Scherer, N.; Marcseková, K.; Posset, T.; Winter, G. New studies on leachables in commercial scale protein drug filling lines using stir bar sorptive extraction coupled with TD-GC-MS and UPLC/ QTOF-MS/MS analytics. Int. J. Pharm. 2019, 555, 404-419. DOI: 10.1016/j.ijpharm.2018.11.033.

9. Tran, J. C.; Doucette, A. A. Cyclic polyamide oligomers extracted from nylon 66 membrane filter disks as a source of contamination in liquid chromatography/mass spectrometry. J. Am. Soc. Mass Spectrom. 2006, 17, 652-656. DOI: 10.1016/j. jasms.2006.01.008.

10. Kiehm, K.; Dressman, J. B. Evaluation of drug adsorption to membrane filters under biowaiver test conditions. Dissolut. Technol. 2008, 15, 13-17. DOI: 10.14227/DT150408P13.

11. K N Lam, P.; Tian, Q.; Ip, M.; Gomersall, C. D. In vitro adsorption of gentamicin and netilmicin by polyacrylonitrile and polyamide 
hemofiltration filters. Antimicrob. Agents Chemother. 2010, 54, 963-965. DOI: 10.1128/AAC.01215-09.

12. Carlson, M.; Thompson, R. D. Analyte loss due to membrane filter adsorption as determined by high-performance liquid chromatography. J. Chromatogr. Sci. 2000, 38, 77-83. DOI: 10.1093/chromsci/38.2.77.

13. Kovalchuk, S. I.; Anikanov, N. A.; Ivanova, O. M.; Ziganshin, R. H.; Govorun, V. M. Bovine serum albumin as a universal suppressor of non-specific peptide binding in vials prior to nano-chromatography coupled mass-spectrometry analysis. Anal. Chim. Acta 2015, 893, 57-64. DOI: 10.1016/j. aca.2015.08.027.

14. Kirkland, J. J. Ultrafast reversed-phase high-performance liquid chromatographic separations: an overview. J. Chromatogr. Sci. 2000, 38, 535-544. DOI: 10.1093/chromsci/38.12.535.

15. Pillai, S. A.; Chobisa, D.; Urimi, D.; Ravindra, N. Filters and filtration: A review of mechanisms that impact cost, product quality and patient safety. J. Pharm. Sci. Res. 2016, 8, 271-278. 